ORIGINAL ARTICLE

\title{
Study of Lipid Profile in Chronic Kidney Disease Patients
}

\author{
RASHID AHMAD ${ }^{1}$, KHALIL ULLAH ${ }^{2}$, GHAZALA SHAHEEN ${ }^{3}$, MUHAMMAD IKRAM SHAH ${ }^{4}$, MUAZZAM FUAAD \\ MUHAMMAD BILAL ${ }^{6}$ \\ ${ }^{1}$ Internal Medicine Assistant Professor Emergency Department, Lady Reading Hospital, Peshawar \\ ${ }^{2}$ Physician Internal Medicine Amjad Kakakhel Clinic, Mardan \\ ${ }^{3}$ Internal Medicine Assistant Professor Pak International Medical College, Peshawar \\ ${ }^{4}$ Assistant Professor Medicine Jinnah Medical College, Peshawar \\ ${ }^{5}$ Assistant Professor Medicine, Rai Medical College, Sargodha \\ ${ }^{6}$ Assistant Professor General Medicine Pak International Medical College, Peshawar \\ Corresponding author:Dr Rashid Ahmad, Email:dr.rashidkmcite@gmail.com,Cell No.+92 3005960865
}

\begin{abstract}
Background and Aim: Premature atherosclerosis and increased prevalence of cardiovascular mortality are significantly associated with chronic kidney disease (CKD). The CKD risk factors contribute to cardiovascular and atherogenesis disease. Anemia, inflammation, vascular calcification, lack of physical activity, lipid disorders, endothelial dysfunction, and oxidative stress are various risk-induced factors for CKD patients. The aim of the present study was to evaluate or assess the lipid profile in chronic kidneys disease.

Place and Duration of Study: Conducted at Medicine department of Lady Reading hospital, Peshawar and Pak International Medical College, Peshawar for duration of six months between November 2020 and April 2021.

Materials and Methods: This cross-sectional study was carried out on 70 patients with chronic kidney disease (CKD) with an age range of 18 and 65 years. The male to female ratio was 1.3:1. A Control group of 70 patients of similar age and sex were enrolled in this study. Lipid profile and collection of blood specimen were managed from both groups were taken. Other parameters such as PPBS, creatinine, FBS, and blood urea results were compared for both groups.

Results: The overall mean age of the study group patients was $42.4 \pm 11.5$ years while the control group's mean age was $51.6 \pm 9.8$ years. The prevalence of CKD patients was high $17(24 \%)$ in the age range of 30-40 years. The prevalence of Dyslipidemia parameters such as High TC, High TG, High VLDL-C, HIGH LDL-C and low HDL-C was $49.8 \%, 66.7 \%, 67 \%, 42.5 \%$, and $72.9 \%$ respectively. Overall dyslipidemia prevalence was $81.7 \%$. Significant decrease in HDL-C while the increase in TG and VLDL-C was reported. On comparing hypertension comorbid conditions with triglyceride, HDL, and VLDL statistical significance was found. SPSS version 24 was used for data analysis.

Conclusion: A significant amount of dyslipidemia is found in CKD patients. As a result, treating dyslipidemia will reduce mortality in CKD patients. Patients with CKD are predisposed to accelerated atherosclerosis, which increases the risk of CVD. The presence of an atherogenic lipid profile in CKD is confirmed by this study.

Keywords: CKD, Lipid Profile, Hypertension, Dyslipidemia
\end{abstract}

\section{INTRODUCTION}

Glomerular filtration rate and abnormal function of kidneys are associated pathophysiological spectrum for chronic kidney disease (CKD) [1]. Cardio-vascular disease (CVD) is mostly caused by chronic kidney disease and risk factors of cardio-metabolism lead to patient morbidity and mortality irrespective of their ethnicity and race [2]. A study conducted on the prevalence of CVD with and without CKD in the United States found $63 \%$ and $5.9 \%$ respectively. The CKD severity is directly related to the CVD prevalence [3]. In dialysis patients, about $45 \%$ of death caused by CVD. Dyslipidemia is a well-known risk factor for CVD. The morbidity and mortality independent predictors of CVD include high-density lipoprotein cholesterol (HDL-C), lowdensity lipoprotein cholesterol (LDL-C), and total cholesterol (TC) as reported by many observers on a largescale basis [4]. CVD pathogenesis in CKD patients is caused by various risk factors among which dyslipidemia is important and modified risk factors [5]. The hypercholesterolemia prevalence varies from $47 \%$ to $79 \%$ in CKD stage 1-2 and CKD stage 3-4respectively [6]. A number of researchers found that hypertriglyceridemia along with decreased HDL-C and increased Very-Low Density Lipoprotein Cholesterol (VLDL-C) in CKD patients leads to abnormal lipid profile $[7,8]$. The risk of CVD and atherosclerosis increase with lipoprotein concentrations and abnormal lipid in CKD patients [9]. Lipo-Protein Lipase(LPL) impaired activity and lipid metabolism involving enzymes of uremic toxin as a direct inhibitor significantly affect elevated triglyceride (TG) levels [10].CKD stages become worsening with dyslipidemia which increased morbidity and mortality caused by cardiovascular disease [11].

Lipoprotein transport abnormalities accompanied with proteinuria cause progressive renal failure. Proteinuria patients might have higher level cholesterol. Typically triglycerides increased serum levels, high level VLDL, low HDL levels, and increased HDL-levels is predominant reflection of dyslipidemia [12]. They cause a selfperpetuating inflammatory response in which macrophages take them up and form foam cells, which are a hallmark of the atherosclerotic process. Endothelial function is also harmed by atherogenic lipoproteins. The resulting arterial narrowing impairs blood supply to various organs [13]. The present study was carried out with an aim to evaluate the lipid profile in Chronic Kidney Diseases.

\section{MATERIALS AND METHODS}

This cross-sectional study was carried out on 70 patients with chronic kidney disease (CKD) with an age range of 18 
and 65 years. A Control group of 70 patients of similar age and sex were enrolled in this study. Lipid profile and collection of blood specimen were managed from both groups were taken. Other parameters such as PPBS, creatinine, FBS, and blood urea results were compared for both groups. Chronic kidney disease 70 consecutive samples were assessed with typical risk factors and lipid profile attending the Medicine department of Lady Reading hospital, Peshawar and Pak International Medical College, Peshawar for duration of six months between November 2020 and April 2021.. Compared to the general population, sample characteristics were derived for each individual. Patients with lowering lipid addiction, renal transplant, and peritoneal dialysis were excluded.

Informed consent was taken from patients diagnosed with CKD and presented to the hospital were enrolled. The onset history, various symptoms, and duration, diet and drug history, and progression were recorded. A sample of overnight fasting was taken and immediately sent to the laboratory. DL concentration changes artifactually after 2 hours of sample collection and within two hours serum separates. Prior to clot retraction, the serum is centrifuged in a centrifugal tube for 5 minutes at 2000 revolutions per minute. Piston pipettes pipetted the clear supernatant serum with dry disposable tips and stored it in vials of the dry thin wall at 4 oC. After 48 hours, samples were analyzed with colorimetric tubes on a biochemistry analyzer.
Parameters

1. S. Cholesterol

2. S. TG

3. $\mathrm{HDL}$

4. LDL

5. VLDL

6. PPBS

7. FBS

8. Creatinine Level
Normal Range

$150-250 \mathrm{mg} / \mathrm{dl}$

$40-160 \mathrm{mg} / \mathrm{dl}$

$35-80 \mathrm{mg} / \mathrm{dl}$

up to $130 \mathrm{mg} / \mathrm{dl}$

$<32 \mathrm{mg} / \mathrm{dl}$

$70-140 \mathrm{mg} / \mathrm{dl}$

$70-110 \mathrm{mg} / \mathrm{dl}$

$0.6-1.3 \mathrm{mg} / \mathrm{dl}$

\section{RESULTS}

The overall mean age of the study group patients was $42.4 \pm 11.5$ years while the control group's mean age was $51.6 \pm 9.8$ years. The prevalence of CKD patients was high
$17(24 \%)$ in the age range of $30-40$ years. The male to female ratio was 1.3:1. The prevalence of Dyslipidemia parameters such as High TC, High TG, High VLDL-C, HIGH LDL-C and low HDL-C was $49.8 \%, 66.7 \%, 67 \%$, $42.5 \%$, and $72.9 \%$ respectively. Overall dyslipidemia prevalence was $81.7 \%$. Significant decrease in HDL-C while the increase in TG and VLDL-C was reported. On comparing hypertension comorbid conditions with triglyceride, HDL, and VLDL statistical significance was found. Table 1 shows the age and gender distribution of the chronic kidney disease patients. Gender distribution of 70 CKD patients is shown in Figure 1.

Table 1. Age and gender distribution of CKD patients $(n=70)$

\begin{tabular}{|c|c|c|c|}
\hline Age (Years) & Frequency $\mathrm{n}(\%)$ & $\begin{array}{l}\text { Male } \\
(\%)\end{array}$ & Female n (\%) \\
\hline $18-30$ & $8(11.4)$ & $5(62.5)$ & $3(37.5)$ \\
\hline $31-40$ & $23(32.9)$ & $11(47.8)$ & $12(52.2)$ \\
\hline $41-50$ & $15(21.4)$ & $9(60)$ & $6(40)$ \\
\hline $51-60$ & $13(18.6)$ & $8(61.5)$ & $5(38.5)$ \\
\hline $61-65$ & $11(15.7)$ & $7(63.6)$ & $4(36.4)$ \\
\hline Total & $100(100)$ & 40 & 30 \\
\hline
\end{tabular}

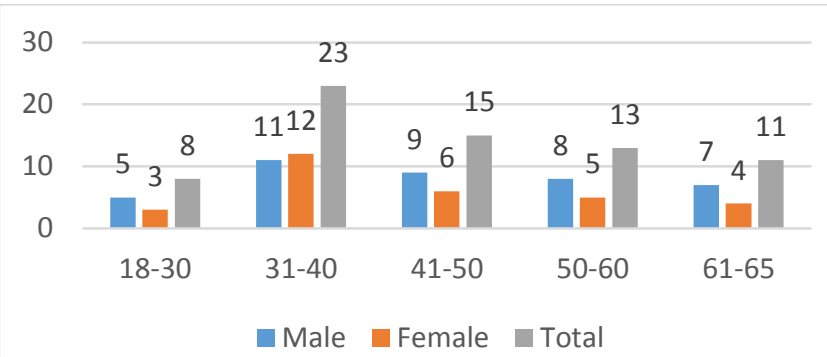

Figure 1. Gender distribution of 70 CKD patients.

Of the 70 chronic kidney disease, male and female patients were $40(57.1 \%)$ and $30(42.9 \%)$ respectively. A high prevalence of CKD was found in the patient's age group between 30 and 40 years. The mean age for males and females was 46.34 and 41.12 years respectively. The biochemical analysis data for both chronic kidney disease and control cases were calculated and are shown in Table 2 and Figure 2.

Table 2. CKD patients and control cases biochemical data $(\mathrm{mg} / \mathrm{dl})$

\begin{tabular}{|l|l|l|l|l|}
\hline Parameters $(\mathrm{mg} / \mathrm{dl})$ & $\begin{array}{l}\text { Control Cases } \mathrm{n}=70 \\
(\text { Mean } \pm \text { SD) }\end{array}$ & $\begin{array}{l}\text { CKD Patients } \mathrm{n}=70 \\
(\text { Mean } \pm \text { SD) }\end{array}$ & *t-value & Significance \\
\hline Serum Creatinine & $0.83 \pm 0.19$ & $6.02 \pm 3.2$ & 14.5 & HS \\
\hline Blood Urea & $23.2 \pm 9.4$ & $141.6 \pm 79.3$ & 10.6 & HS \\
\hline
\end{tabular}

*t-test student $t$-test $\mathrm{p}<0.001$ highly significant, $\mathrm{HS}=$ highly significant

Considerable and higher the difference was observed in the blood urea and creatinine serum respectively with mean value and statistical significance $(p<0.001)$. The lipid profile of control and CKD cases was compared in Table/Figure-3. The cholesterol values were $195.6 \pm 58.5$ $\mathrm{mg} / \mathrm{dl}$ and $161.9 \pm 43.8 \mathrm{mg} / \mathrm{dl}$ in chronic kidney patients and control group $(p<0.05)$ and statistical significance was observed. However, no significance was observed in the difference regardless of high differences in Triglycerides values which were $141 \pm 63.8 \mathrm{mg} / \mathrm{dl}$ and $168.9 \pm 101.9$ in control and CKD cases respectively. HDL and VLDL values were $38.6 \pm 8.9 \mathrm{mg} / \mathrm{dl}$ and $28.9 \pm 14.32 \mathrm{mg} / \mathrm{dl}$ in control cases while $38.52 \pm 9.04 \mathrm{mg} / \mathrm{dl}$ and $33.82 \pm 20.54 \mathrm{mg} / \mathrm{dl}$ in chronic kidney disease cases respectively. In both control and CKD cases, HDL levels were similar and VLDL levels were high in chronic kidney patients. The TG level were 141.2 263.51 in control cases while in CKD patients, it was 170.23 \pm 101.9 .

Table/Figure-4 demonstrates the lipid profile of male and female chronic kidney disease patients. The increased mean values of TG, LDL, and VLDL were reported in male 
patients while HDL levels decreased. On the other hand, LDL levels increased in females patients.

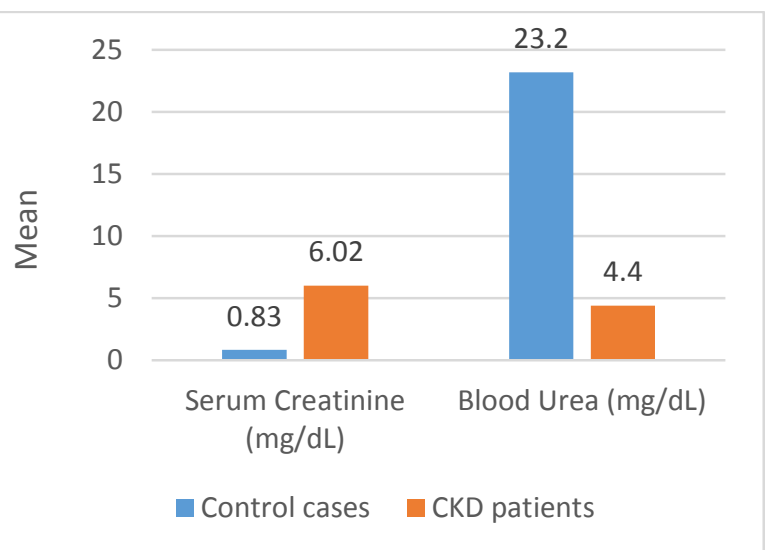

Figure 2. Serum Creatinine and Blood urea analysis of both Control and CKD cases

Table 3. CKD and Control patients' lipid profile.

\begin{tabular}{|l|l|l|l|l|}
\hline Parameter $(\mathrm{mg} / \mathrm{dl})$ & $\begin{array}{l}\text { Control Cases } \\
\mathrm{N}=70\end{array}$ & $\begin{array}{l}\text { CKD Cases } \\
\mathrm{N}=70\end{array}$ & t-value & Significance \\
\hline Cholesterol & $161.9 \pm 43.8$ & $195.6 \pm 58.5$ & 3.381 & $\mathrm{~S}(0.0011)$ \\
\hline HDL & $38.6 \pm 8.9$ & $38.52 \pm 9.04$ & 1.72 & $\mathrm{NS}$ \\
\hline LDL & $101.9 \pm 42.81$ & $121.9 \pm 57.2$ & 0.032 & $\mathrm{~S}(0.05)$ \\
\hline VLDL & $28.9 \pm 14.32$ & $33.82 \pm 20.54$ & 1.943 & $\mathrm{NS}$ \\
\hline TG & $141.2 \pm 63.51$ & $170.23 \pm 101.9$ & 1.68 & $\mathrm{NS}$ \\
\hline
\end{tabular}

Table 4. Lipid profile in male and female patients $(\mathrm{mg} / \mathrm{dl})$

\begin{tabular}{|l|l|l|l|l|}
\hline Parameter $(\mathrm{mg} / \mathrm{dl})$ & Male & Females & t-value & Significance \\
\hline & $\mathrm{N}=40($ Mean $\pm \mathrm{SD})$ & $\mathrm{N}=30($ Mean \pm SD) & & \\
\hline Cholesterol & $198.9 \pm 56.45$ & $194.2 \pm 62.56$ & 0.253 & NS \\
\hline HDL & $38.39 \pm 9.87$ & $38.63 \pm 9.58$ & 1.134 & NS \\
\hline \hline LDL & $119.4 \pm 57.18$ & $125.83 \pm 61.6$ & 0.672 & NS \\
\hline VLDL & $37.3 \pm 19.6$ & $29.85 \pm 21.56$ & 0.260 & NS \\
\hline TG & $181.9 \pm 101.4$ & $147.2 \pm 105.85$ & 1.042 & NS \\
\hline
\end{tabular}

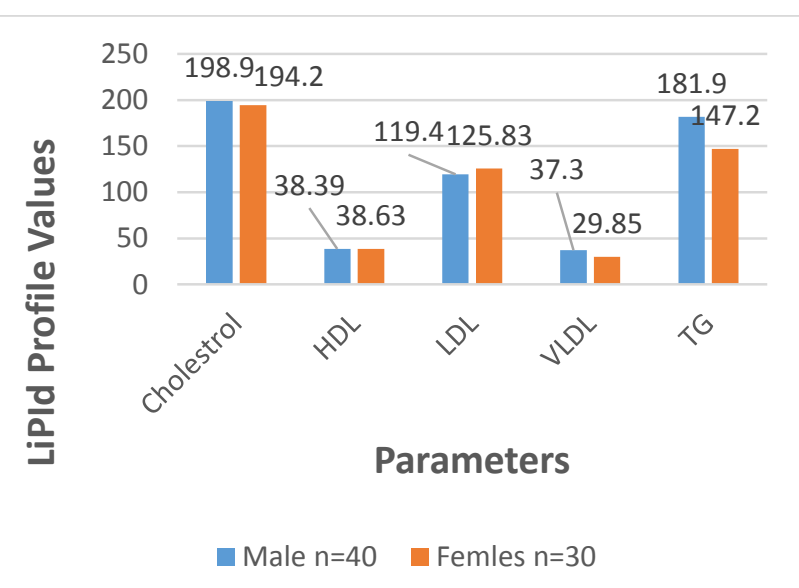

Figure 4. Lipid profile in male and female patients $(\mathrm{mg} / \mathrm{dl})$

\section{DISCUSSION}

The present study revealed the alteration of lipid profile levels in chronic kidney disease patients and control cases.

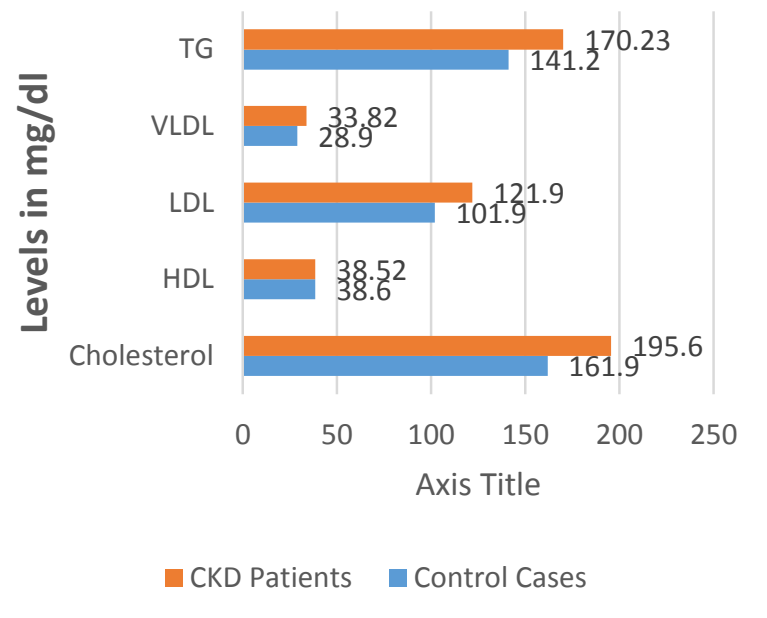

Figure 3. CKD and Control patients' lipid profile.

Another study [14] reported elevation in the levels of serum creatinine levels, VLDL, and $\beta$ HDL. However, the HDL level was reduced. Proteinuria levels were directly proportional to triglycerides level and nephrotic syndrome in primary patients. Another study found higher triglyceride serum levels and decreased VLDL and chylomicrons in chronic kidney patients [15]. In chronic kidney disease patients, the most common plasma was found in hypertriglyceridemia [16]. Cholesterol concentration and HDL were found lower in CKD patients. Total synthetic rate and catabolic fractional rate were reported lower with HDL levels. Another study carried out on cholesterol levels in CKD patients found no significant changes [17]. VLDL levels were found higher in CKD patients [18].

Another study found high levels of LDL and cholesterol were reported in chronic kidney disease patients as compared to control cases. Statistical significance was found in these two levels. Higher triglycerides level was found in CKD and control group but no significant was reported. The VLDL and HDL-like lipoprotein fractions levels were within the standard limit and the mean was found not significant [19]. Another study 
compared lipid profiles in chronic kidney disease and control group but found elevated serum triglyceride levels with abnormalities in lipid pathogenesis involving removal of defective material from circulation. The glycerides lead to hypertriglyceridemia used by triglycerides clearance as in composition alteration. Triglyceride lipids and lipoprotein lipase activities are reduced which remove triglycerides [20].

Nephrotic syndrome and other factors cause renal failures in patients with lipid abnormalities [21]. It was found that renal impairment severity is proportional to the elevated HDL cholesteroland hypertriglyceridemia prevalence. However, the study also reported the higher triglycerides in diabetic patients along with exacerbated abnormalities of lipid in diabetes with lower HDL. Another study compared lipid profile and CKD with diabetic and non-diabetic patients [22]. Elevated triglycerides, VLDL and LDL was found in patients of chronic kidney disease when diabetic and non-diabetic lipid profile was compared. The study didn't find any correlation among diabetic and non-diabetic patients besides lipoprotein fractions elevation [23].The statistical significance of lipid profile parameters matched another study [24] study statistical value. In our study, lipoprotein fractions were found within normal range and significant. Carbohydrate low calories and polyunsaturated fatty acid high intake in most people's diet could be the attributes for these lipoprotein parameters and regional behavior. Hence, in our population just like the Western population lower degree of hypertriglyceridemia was reported. The high intake of fatty acid (polyunsaturated) in the form of dietary patterns could be the cause for these factors. However, because the study group in this study was heterogeneous, the data collected should have likely included a larger group.

\section{CONCLUSION}

A significant amount of dyslipidemia is found in CKD patients. As a result, treating dyslipidemia will reduce mortality in CKD patients. Patients with CKD are predisposed to accelerated atherosclerosis, which increases the risk of CVD. The presence of an atherogenic lipid profile in CKD is confirmed by this study.

\section{REFERENCES}

1. Zhang YB, Sheng LT, Wei W, Guo H, Yang H, Min X, Guo K, Yang K, Zhang $X, \mathrm{He} M, \mathrm{Wu} T$. Association of blood lipid profile with incident chronic kidney disease: a Mendelian randomization study. Atherosclerosis. 2020 May 1;300:19-25.

2. Rysz J, Gluba-Brzózka A, Rysz-Górzyńska M, Franczyk B. The role and function of HDL in patients with chronic kidney disease and the risk of cardiovascular disease. International journal of molecular sciences. 2020 Jan;21(2):601.

3. GBD 2017 Disease and Injury Incidence and Prevalence Collaborators, Global, regional, and national incidence, prevalence, and years lived with disability for 354 diseases and injuries for 195 countries and territories, 1990-2017: a systematic analysis for the Global Burden of Disease Study 2017, Lancet 392 (2018) 17891858.

4. GBD 2017 Causes of Death Collaborators, Global, regional, and national age-sexspecific mortality for 282 causes of death in 195 countries and territories, 1980- 2017: a systematic analysis for the Global Burden of Disease Study 2017, Lancet 392 (2018) 17361788 .

5. A.C. Webster, E.V. Nagler, R.L. Morton, P. Masson, Chronic kidney disease, Lancet 389 (2017) 1238-1252.
6. S. Merscher, C.E. Pedigo, A.J. Mendez, Metabolism, energetics, and lipid biology in the podocyte - cellular cholesterol-mediated glomerular injury, Front. Endocrinol. (Lausanne) 5 (2014) 169.

7. B. Bowe, Y. Xie, H. Xian, S. Balasubramanian, Z. Al-Aly, Low levels of high-density lipoprotein cholesterol increase the risk of incident kidney disease and its progression, Kidney Int. 89 (2016) 886-896.

8. Kajani, S.; Curley, S.; McGillicuddy, F.C. Unravelling HDL-Looking beyond the Cholesterol Surface to the Quality Within. IJMS 2018, 19, 1971. [CrossRef] [PubMed]

9. 56. van der Stoep, M.; Korporaal, S.J.A.; Van Eck, M. High-density lipoprotein as a modulator of platelet and coagulation responses. Cardiovasc. Res. 2014, 103, 362-371.

10. Bardagjy, A.S.; Steinberg, F.M. Relationship between HDL Functional Characteristics and Cardiovascular Health and Potential Impact of Dietary Patterns: A Narrative Review. Nutrients 2019, 11, 1231. [CrossRef] [PubMed]

11. Chiesa, S.T.; Charakida, M. High-Density Lipoprotein Function and Dysfunction in Health and Disease. Cardiovasc. Drug Ther. 2019, 33, 207-219. [CrossRef] [PubMed]

12. Honda, H.; Hirano, T.; Ueda, M.; Kojima, S.; Mashiba, S.; Hayase, Y.; Michihata, T.; Shibata, T. High-Density Lipoprotein Subfractions and Their Oxidized Subfraction Particles in Patients with Chronic Kidney Disease. J. Atheroscler. Thromb. 2016, 23, 81-94. [CrossRef] [PubMed]

13. Tolle, M.; Huang, T.; Schuchardt, M.; Jankowski, V.; Prufer, N.; Jankowski, J.; Tietge, U.J.; Zidek, W.; van der Giet, M. High-density lipoprotein loses its anti-inflammatory capacity by accumulation of pro-inflammatory-serum amyloid A. Cardiovasc. Res. 2012, 94, 154162. [CrossRef]

14. Cwikli 'nska, A.; Cackowska, M.; Wieczorek, E.; Kr' ól, E.; Kowalski, R.; Kuchta, A.; Kortas-Stempak, B.; Gliwi 'nska, A.; D a abkowski, K.; Zieli 'nska, J.; et al. Progression of Chronic Kidney Disease Affects HDL Impact on Lipoprotein Lipase (LPL)-Mediated VLDL Lipolysis Efficiency. Kidney Blood Press. Res. 2018, 43, 970-978. [CrossRef]

15. Wieczorek, E.; Cwiklinska, A.; Cackowska, M.; Kuchta, A.; Krol, E.; Kortas-Stempak, B.; Gliwinska, A.; Dabkowski, K.; Jankowski, M. The impact of HDL concentration on efficiency of lipoprotein lipase (LPL)mediated VLDL lipolysis in patients with chronic kidney disease. Atherosclerosis 2017, 263, e210. [CrossRef]

16. Huang, Y.; DiDonato, J.A.; Levison, B.S.; Schmitt, D.; Li, L.; Wu, Y.; Buffa, J.; Kim, T.; Gerstenecker, G.S.; Gu, X.; et al. An abundant dysfunctional apolipoprotein 63 in human atheroma. Nat. Med. 2014, 20, 193-203. [CrossRef]

17. Shao, B.; Tang, C.; Heinecke, J.W.; Oram, J.F. Oxidation of apolipoprotein $A-I$ by myeloperoxidase impairs the initial interactions with $\mathrm{ABCA} 1$ required for signaling and cholesterol export. J. Lipid Res. 2010, 51, 1849-1858. [CrossRef]

18. Vaziri, N.D. HDL abnormalities in nephrotic syndrome and chronic kidney disease. Nat. Rev. Nephrol. 2016, 12, 37-47.

19. Li, Y.; Zhao, M.; He, D.; Zhao, X.; Zhang, W.; Wei, L.; Huang, E.; Ji, L.; Zhang, M.; Willard, B.; et al. HDL in diabetic nephropathy has less effect in endothelial repairing than diabetes without complications. Lipids Health Dis. 2016, 15, 76. [CrossRef] [PubMed]

20. Thompson, M.; Ray, U.; Yu, R.; Hudspeth, A.; Smillie, M.; Jordan, N.; Bartle, J. Kidney Function as a Determinant of HDL and Triglyceride Concentrations in the Australian Population. J. Clin. Med. 2016, 5, 35. [CrossRef] [PubMed]

21. Kopecky, C.; Haidinger, M.; Birner-Grünberger, R.; Darnhofer, B.; Kaltenecker, C.C.; Marsche, G.; Holzer, M.; Weichhart, T.; Antlanger, M.; Kovarik, J.J.; et al. Restoration of Renal Function Does Not Correct Impairment of Uremic HDL Properties. JASN 2015, 26, 565575. [CrossRef] [PubMed]

22. Shah, A.S.; Tan, L.; Long, J.L.; Davidson, W.S. Proteomic diversity of high density lipoproteins: Our emerging understanding of its importance in lipid transport and beyond. J. Lipid Res. 2013, 54, 2575-2585. [CrossRef] [PubMed]

23. De Pasquale, C.G.; Arnolda, L.F.; Doyle, I.R.; Aylward, P.E.; Chew, D.P.; Bersten, A.D. Plasma surfactant protein-B: A novel biomarker in chronic heart failure. Circulation 2004, 110, 1091-1096. [CrossRef]

24. Otocka-Kmiecik, A.; Mikhailidis, D.P.; Nicholls, S.J.; Davidson, M.; Rysz, J.; Banach, M. Dysfunctional HDL: A novel important diagnostic and therapeutic target in cardiovascular disease? Prog. Lipid Res. 2012, 51, 314-324. [CrossRef] 82. Khera, A.V.; Rader, D.J. Future therapeutic directions in reverse cholesterol transport. Curr. Atheroscler. Rep. 2010, 12, 73-81. [CrossRef] 